\title{
An adaptive method for water pipeline leak localization
}

\author{
Shun Na, Jinhong Liu, Qi Li, Yang Liu, Yong Tie* \\ College of Electronic Information Engineering, Inner Mongolia University, Hohhot, 010021, China \\ *Corresponding author: nashunimu@163.com.
}

Keywords: Leak localization, neural network, generalized cross correlation, adaptive filter.

\begin{abstract}
A common method for leak localization in water pipelines is the use of cross correlation on two measured acoustic signals located on either side of a leak. However, in practice, leak signals are inevitably corrupted with non-leak sounds and noise. This is due to the complicated pipeline environment that makes time delay estimation between the sensors inaccurate. In addition, time delay errors further affect leak localization accuracy, and then reduce the reliability of the system. In this paper, a new adaptive leak detection method that combines BP neural networks with GCC (generalized cross correlation) is proposed. The new proposed method can discriminate the leak signal from non-leak acoustic sources and noise, and improve leak localization accuracy. Simulation results indicates that the proposed leak detection method can be used to localize a leak in a buried water pipeline and achieved a correct detection rate of $92.5 \%$.
\end{abstract}

\section{Introduction}

The status of water resources not only directly affects the national economy and the people's livelihood but also restricts economic and social development. With a shortage of water resources in the world, the importance of protecting water supplies to cities is increasingly critical. Thus, more attention must be paid to water conservation. It has been estimated that within the Asian region, at least $25 \%$ of the water supply is being lost within the water distribution system [1]. Pipelines play an irreplaceable role in managing water supplies because of their simple transmission process and large transmission capacity [2]. Therefore, it is extremely important to develop a timely and accurate leak localization method for use in water supply pipelines. After decades of development, the key techniques used for the reduction of pipeline leakage are many and varied. Methods that have appeared successively include listening rods, correlators, ground penetrating radar (GPR), and robots. Although traditional water leakage location methods are easy to operate, such as in the case of a listening stick equipped with an electronic magnifying leak detector, their stability and accuracy are poor. These traditional methods are more dependent on manpower and are no longer suitable for modern cities. A generalized correlation method has been used recently for modern water leakage location. This method selects a corresponding correlation function for different environments, and a weighted correction is conducted in the frequency domain, thereby making the experimental estimation more accurate. There has been a significant improvement in accuracy, but this method cannot be applied to complex natural environments or over wide ranges of water leakage locations. Even so, due to the high accuracy and the simple algorithm, this correlation method has been shown to be effective $[3,4]$ and is commonly used in the water industry. As a result, the cross-correlation method has been used for time delay estimation, and this is a crucial factor that can affect the accuracy of water leak localization.

To decrease errors that occur in the time delay estimation and improve the localization accuracy of the algorithm, a method using a back propagation (BP) neural network that is based on the error back propagation algorithm is proposed [5]. The back propagation (BP) neural network consists of input layers, hidden layers, and output layers. The propagation algorithm is a supervised learning algorithm. It inputs learning sample firstly, and then improves the network weights and thresholds constantly with repeated adjustment training. In order to decrease the error accuracy of the output vector, the back-propagation algorithm adjust the target vector by requirements. Finally, network 
training is completed when the sum square errors of the output layer is less than the threshold. The essence of a back propagation neural network (BPNN) is to minimize the change in weights by using a gradient descent method to finally attain minimal error.

In this paper, we propose a novel water pipeline leak localization scheme, which is a reasonable combination of BP neural network and the generalized cross correlation (GCC). First, we can use the adaptive filter based on the characteristics of BPNN to remove the in-band noise of the leaked signal. Then the generalized cross correlation (GCC) is applied to the time difference of arrival (TDOA) estimation to achieve precise localization of the water leakage point after multi-resolution decomposition and delay superposition. A precise water pipeline leak position can be obtained by the above method. In addition, the new method is not only more practical but also can achieve high accuracy, low power consumption, and a wide range of leak detection. Such characteristics are validated by both theoretical analysis and simulation results.

The rest of this paper is organized as follows. The leak localization algorithm based on BP neural network (BPNN) training and generalized cross-correlation (GCC) time difference of arrival (TDOA) estimation are introduced in Section II. The simulation results of the proposed method are shown in Section III. Finally, conclusions are drawn in Section IV.

\section{Principles of leak localization in buried water pipe}

\subsection{The model of leak localization}

Vibration or acoustic signals are measured using accelerometers at three access points, with the distribution of sensors being an equal distance, $d$, and a suspected leak on either side of the location. Fig. 1 depicts the new method for the measurement of a water leak in a buried water pipe. Access points (normally a fire hydrant) [6] where a sensor can be attached are located on each side of the leak at distances $\mathrm{d} 1$ and $\mathrm{d} 2$.

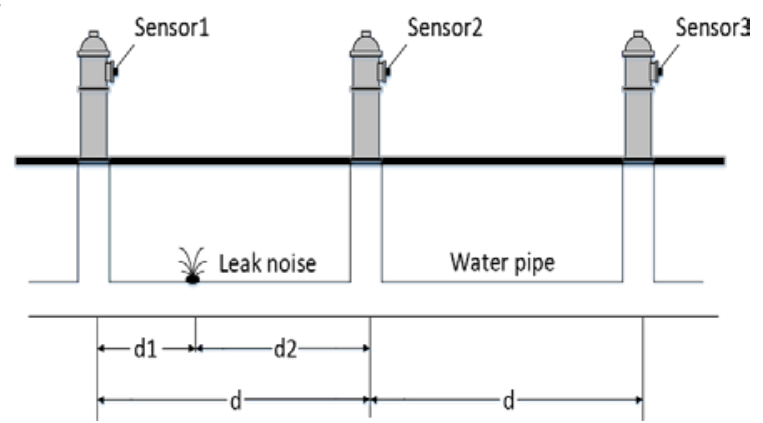

Fig. 1. Schematic of a pipe with a leak bracketed by three sensors.

In contrast with traditional localization methods that use two sensors, the system can calculate the speed, $v$, of the leak signals with a change of the pipeline using the following equation:

$$
v=d / D_{23}
$$

Where $D_{23}$ is the lag of time between sensor 2 and sensor 3. As shown in Fig 1, the time delay is related to the locations of the sensors according to the following equations:

$$
\left\{\begin{array}{c}
d=d_{1}+d_{2} \\
D_{21} \times v=d_{2}-d_{1}
\end{array}\right.
$$

By substituting $v$ into Equation (2), the position of the leak relative to sensor 1 and sensor 2 is found to be 


$$
\left\{\begin{array}{l}
d_{1}=\frac{d \times\left(D_{23}-D_{21}\right)}{2 D_{23}} \\
d_{2}=\frac{d \times\left(D_{23}+D_{21}\right)}{2 D_{23}}
\end{array}\right.
$$

\subsection{The key techniques of the localization method}

The key techniques used in this system include the BPNN and the GCC. A BPNN is a type of back-propagation feed-forward network whose topological structure is depicted in Fig. 2. The backpropagation algorithm is a supervised learning algorithm, which trains a neural network using a gradient descent algorithm in which the mean square errors between the network's output and the desired output are minimized [8]. The network is composed of input layers, hidden layers, and output layers, wherein each layer contains multiple nodes as separate inputs to achieve full connectivity between the layers. The GCC time delay estimation method being proposed here is based on signal pre-processing, and this can reduce the error in the lower bound of the Cramer Rao (CRB) [7]. But this method relies on a prior knowledge of statistics, which is not easy to achieve in a complicated environment, such as in underground water pipes. To overcome this shortcoming, an improved algorithm combining BPNN with GCC is proposed that will provide a self-adaptive leak localization method.

Some well-known advantages of BPNN include strong non-linear mapping ability, flexible network structure, andhe numbers of intermediate layers and neurons in each layer is depended on the circumstances. Furthermore, BP neural network has preliminary adaptive and self-organization capabilities, since BPNN can store a large number of input-output mappings which eliminates storing a large number of mappings in advance. Thereby, constructing an adaptive filtering system based on the BPNN is possible. It can be applied to water pipeline leak localization system for reducing noise, which lack a large number of prior conditions. In the base of this, improving accuracy localization of the leakage signals further is feasible.

The parameter settings are as follows:

$M, I, J$ is the item count of the input layer, the hidden layer, and the output layer, respectively.

$x_{m}, k_{i}, y_{j}$ is the m-th input unit, the $\mathrm{i}$-th hidden unit, and the $\mathrm{j}$-th output unit, respectively.

$\bar{w}_{m i}, \bar{w}_{i j}$ is the weight value between layers of network, respectively.

$u, v$ is the iterative number of each layer.

$f()$ is the sigmoid function.

$g()$ is the transfer function of the output layer.

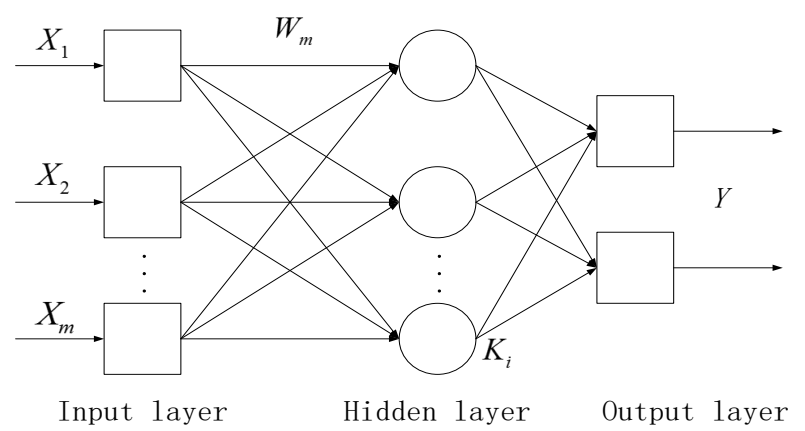

Fig. 2. Topology structure of a three-layer BPNN.

As Fig. 2 shows, vectors are applied to each layer of the network. The actual output and desired output are

$$
\begin{aligned}
& Y(n)=\left[v_{J}^{1}, v_{J}^{2}, \ldots, v_{J}^{J}\right] \\
& d(n)=\left[d_{1}, d_{2}, \ldots, d_{J}\right]
\end{aligned}
$$


The nth iteration error is given by

$$
e(n)=d_{j}(n)-Y_{j}(n)
$$

According to the energy definition, the error energy is

$$
e(n)=\frac{1}{2} \sum_{j=1}^{J} e_{j}^{2}(n)
$$

where $e_{j}(n)$ refers to the error of the $j$-th output unit. In the process of error back propagation, the weights are adjusted along the gradient direction of the error, and the calculation of the gradient obtained by the chain rule of the differential is

$$
\frac{\partial e(n)}{\partial \omega_{i j}(n)}=\frac{\partial e(n)}{\partial e_{j}(n)} \cdot \frac{\partial e_{j}(n)}{\partial v_{J}^{j}(n)} \cdot \frac{\partial v_{J}^{j}(n)}{\partial u_{J}^{j}(n)} \cdot \frac{\partial u_{J}^{j}(n)}{\partial \omega_{i j}(n)}
$$

The local gradient is defined to be

$$
\delta_{J}^{j}=\frac{\partial e(n)}{\partial u_{J}^{j}(n)}=-\frac{\partial e(n)}{\partial e_{j}(n)} \cdot \frac{\partial e_{j}(n)}{\partial v_{J}^{j}(n)} \cdot \frac{\partial v_{J}^{j}(n)}{\partial u_{J}^{j}(n)}=e_{j}(n) g^{\prime}\left(u_{J}^{j}(n)\right)
$$

Because the transfer function of the output layer is linear, the derivative can be a constant, $\mathrm{k}$, and its learning rate is $\eta$. By substituting $\mathrm{k}$ and $\eta$ into Equation (9), the correction of the neuron weight is found to be:

$$
\Delta \omega_{i j}(n)=k \eta e_{j}(n) v_{I}^{i}(n)
$$

The time difference of arrival (TDOA) estimation method based on the generalized cross correlation (GCC) is a key technology in the water pipeline leak localization. Its principle is to weight the signal before the cross-correlation operation. Therefore, the TDOA estimation performance of the correlation operation is improved, and the detection accuracy of the system is improved through weighted processing.

According to Fig.1, the cross-correlation function of the two received signals can be expressed as

$$
R_{x_{1} x_{2}}(\tau)=E\left[x_{1}(n-\tau) x_{2}(n)\right]=R_{s s}\left(\tau-\frac{d_{1}}{v T_{s}}+\frac{d_{2}}{v T_{s}}\right)+R_{s n_{1}}\left(\tau-\frac{d_{1}}{v T_{s}}\right)+R_{s n_{2}}\left(\tau+\frac{d_{2}}{v T_{s}}\right)+R_{n_{1} n_{2}}(\tau)
$$

Where $x_{1}(n)$ and $x_{2}(n)$ are received signals, $T_{s}$ represents the sampling period. A generalized cross-correlation function is obtained by weighting and filtering $R_{x_{1} x_{2}}(\tau)$, which can be expressed as

$$
R_{y_{1} y_{2}}(\tau)=F^{-1}\left[G_{y_{1} y_{2}}(w)\right]=\alpha^{-1}\left[H(w) G_{x_{1} x_{2}}(w)\right]
$$

Where $H(\omega)=H_{2}(\omega) H_{1}^{*}(\omega)$ denotes generalized cross correlation weighting function, and $G_{y_{1} y_{2}}(\omega)$ denotes generalized power spectrum function, $F$ represents Fourier transform. When $\tau=\left(d_{2}-d_{1}\right) / v T_{s}, R_{y_{1} y_{2}}(\tau)$ obtains the maximum value. In addition, the two received signals have the greatest correlation. According to $\tau$ and Equation (1), the time difference of arrival (TDOA) value can be represented as

$$
\hat{D}=\frac{d_{2}-d_{1}}{v}=\tau \times T_{s}
$$

\section{Simulations of the adaptive system}

The overall idea of the proposed leak location method is that the GCC time difference of arrival (TDOA) estimation is accurate. However, a large amount of prior knowledge and statistical 
characteristics are needed to determine the use of window functions. This is not easy to implement in an under-ground water leakage detection system. Because the environment of the underground leakage pipeline is complex, and the amount of data acquired is small, the BPNN-based positioning method combined with the GCC can overcome this defect and realize an adaptive pipeline leakage location system.

The experimental object used for this system was a cast iron pipe. In this environment, sensors with a sampling rate of $100 \mathrm{ksps}$ were used. The waveform of the leak signals from the sensors on both sides of pipeline are shown in Fig. 3 (a) and Fig. 3 (b).

The simulation environment is the same as in Fig. 3. The frequency spectrum of the leak signals from the sensors on both sides of pipeline are shown in Fig. 4 (a) and Fig. 4 (b).

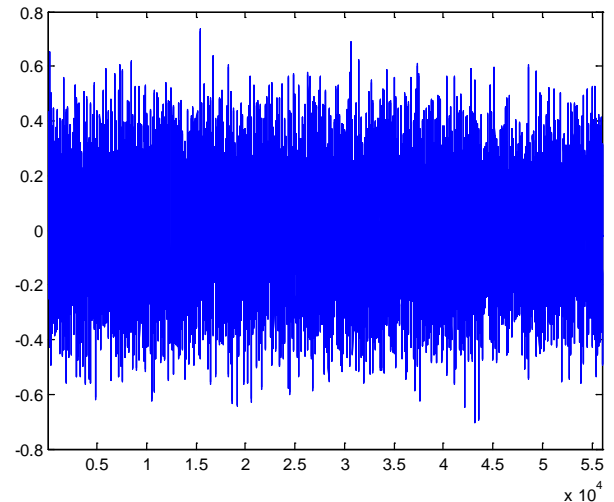

(a)

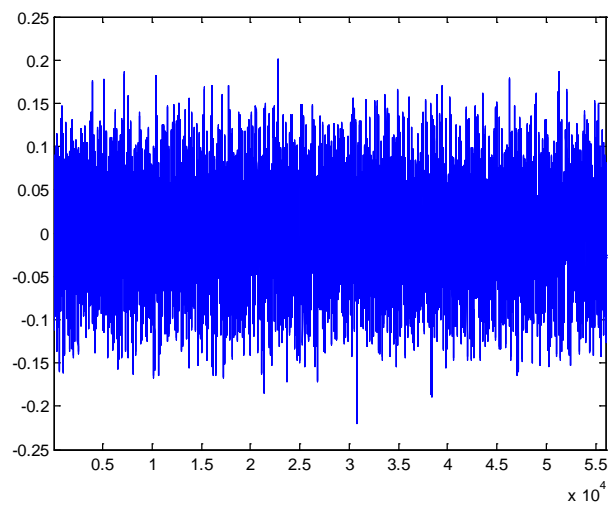

(b)

Fig. 3. The waveform of the two sensors. (a) The original time domain signal of sensor1, (b) The original time domain signal of sensor2.

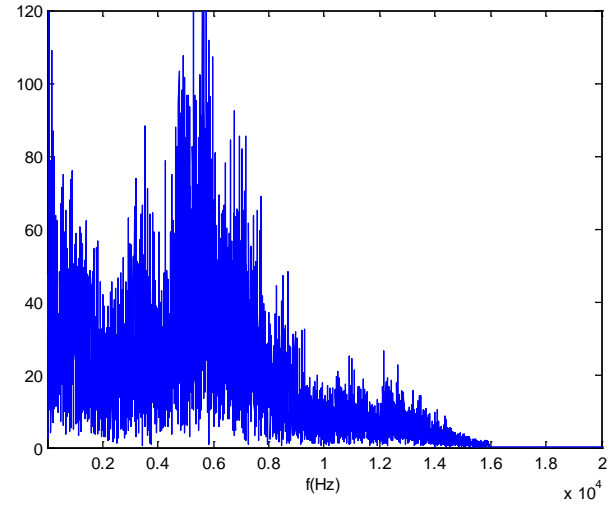

(a)

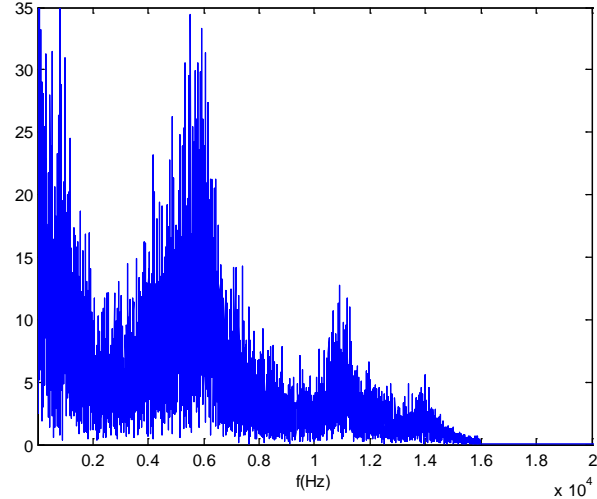

(b)

Fig. 4. The frequency spectrum of the two sensors. (a) The original frequency domain signal of sensor1, (b) The original frequency domain signal of sensor2.

The simulation environment is the same as in Fig. 3. The analysis of the leakage signal took into account that the inflow of gas into the insoluble gas in the pipe stream created bubbles that caused random changes in the frequency spectrum as the bubble moved from the flow to the leakage point, and the leakage was mainly concentrated in the low frequency band [10]. Therefore, the leakage signal was filtered out of the out-of-band noise using a band-pass filter with a filter frequency of $500-5000 \mathrm{~Hz}$, thereby improving the accuracy of the time difference of arrival (TDOA) estimation [11]. The waveform result of the band-pass filter of the leakage signal is shown in Fig. 5 (a) and Fig. 5 (b).

The simulation environment is the same as in Fig. 3. The leakage signal was filtered out of the out-of-band noise using a band-pass filter with a filter frequency of 500-5000 Hz. The frequency spectrum result of the band-pass filter of the leakage signal is shown in Fig. 6 (a) and Fig. 6 (b). 


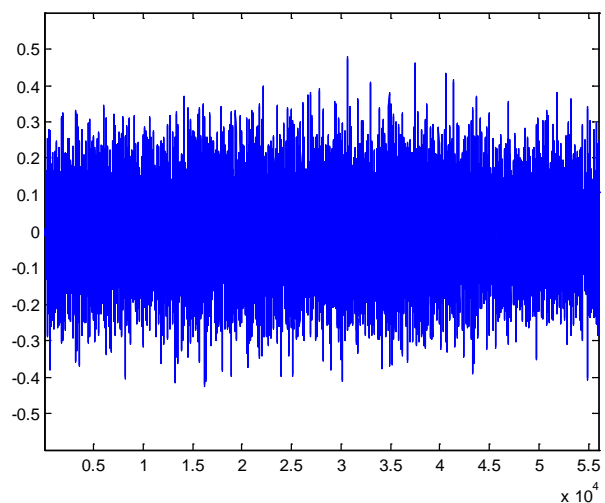

(a)

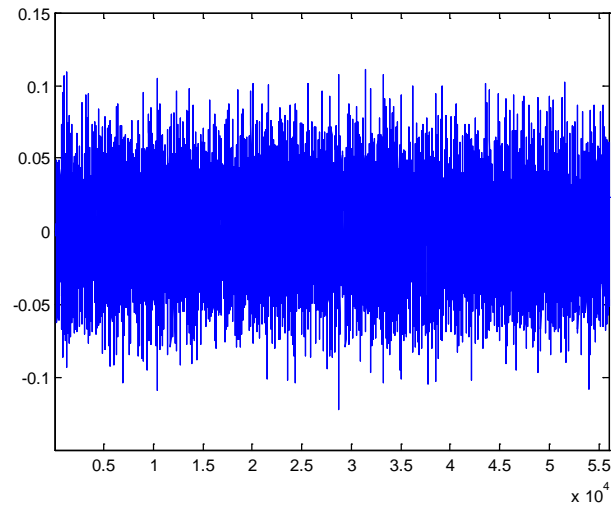

(b)

Fig. 5. The waveform of the sensors after band-pass filtering. (a) Time domain signal of sensor1 after band-pass filtered, (b) Time domain signal of sensor2 after band-pass filtered.

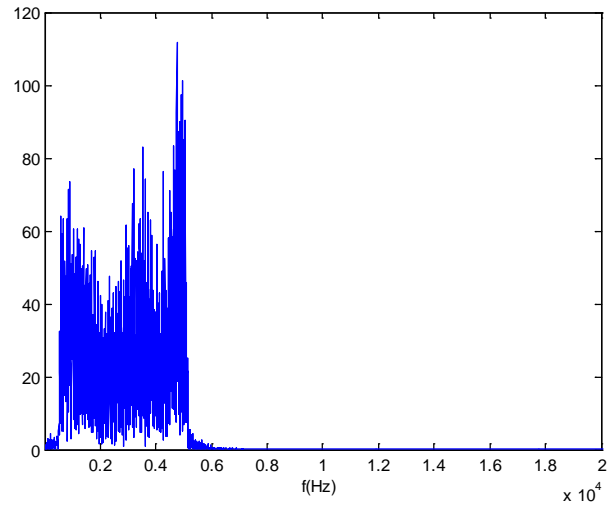

(a)

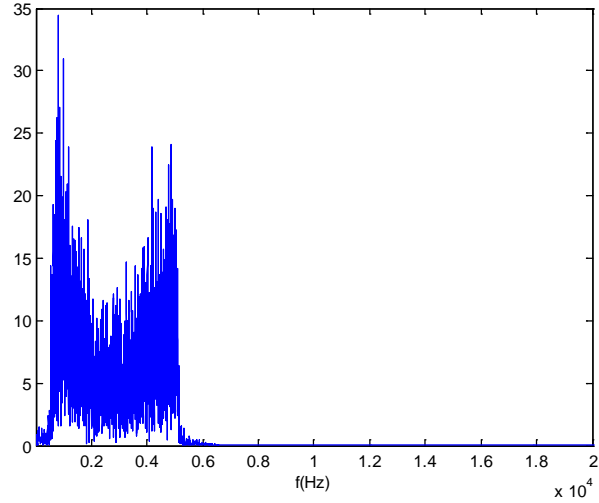

(b)

Fig. 6. The frequency spectrum of the sensors after band-pass filtering. (a) Frequency domain signal of sensor1 after band-pass filtered, (b) Frequency domain signal of sensor2 after band-pass filtered.

After the band-pass filter was used to filter out-of-band noise, noise was also present in the main frequency band where the leakage signal was concentrated. The in-band noise was filtered out using a BPNN filter. The next step was the establishment of the BPNN to discriminate the leak signal from non-leak acoustic sources adaptively. The process of the establishment of the neural network model for time delay estimation was divided into two phases: the first phase was the learning process, and the second phase was the work process [12].

(1) Sample data were selected to construct training samples.

(2) Sample data was pre-processed. Before neural network prediction, the raw data was normalized so that its data specification was between $[-1,1]$.

(3) The signal sampling sequence with a high signal to noise ratio (SNR) was chosen as the desired vector, and then the signal was measured in the actual situation, whose sampling sequence was chosen as the input vector.

(4) The BPNN was realized using a three-layer model, whose number of input nodes, hidden nodes, and output nodes were 1,17 , and 1 , respectively. The transfer function of the hidden layer and the output layer were the Tansig Function and the Purelin Function, respectively [13].

(5) Before training the network, the training parameters settings were set as follows: net.trainParam.epochs $=10000$, net.trainParam.goal $=0.05$, bpnet.trainParam. $\operatorname{lr}=0.01$, and so on .

After training the network, the network was used to discriminate the leak signal from the nonleak acoustic sources adaptively, and then the time delay was estimated with GCC. The graphs of the training result and the GCC function are depicted in Fig 7 (a) and Fig 7 (b). 


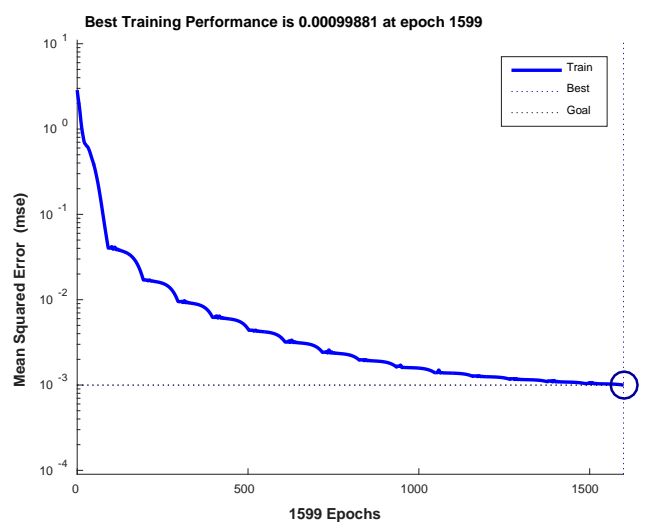

(a)

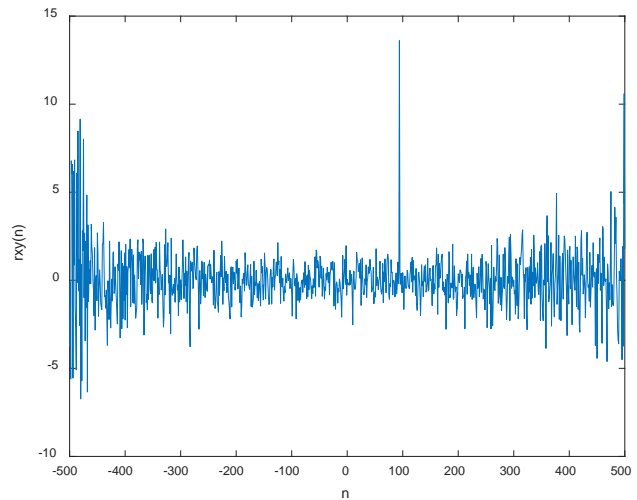

(b)

Fig. 7. Network training performance and the graph of GCC with an SNR of 10dB. (a) Training results of the neural network, (b) GCC function graph.

Table 1 shows the localization results of the two methods. It is clear that the localization precision of the adaptive method was higher than that of traditional cross correlation. This was because the network adaptively changed its weights and bias, thus decreasing the influence of random noise caused by a complicated environment $[14,15]$ and improving the leak localization accuracy by $5.03 \%$.

Table.1 Leak Location Results of the Two Methods.

\begin{tabular}{|c|c|c|c|c|}
\hline $\begin{array}{c}\text { Real distance } \\
(\mathrm{d} 1+\mathrm{d} 2)\end{array}$ & $\begin{array}{c}\text { Traditional method } \\
\mathrm{d} 1(\mathrm{~m})\end{array}$ & $\begin{array}{c}\text { Error } \\
(\%)\end{array}$ & $\begin{array}{c}\text { Adaptive } \\
\text { method } \\
\mathrm{d} 1(\mathrm{~m})\end{array}$ & $\begin{array}{c}\text { Error } \\
(\%)\end{array}$ \\
\hline $3+1$ & 3.47 & 15.67 & 2.72 & 9.33 \\
\hline $5+1$ & 5.71 & 14.20 & 5.41 & 8.20 \\
\hline $6+3$ & 5.52 & 8.67 & 6.13 & 2.16 \\
\hline $7+1$ & 8.06 & 15.14 & 6.38 & 8.86 \\
\hline $10+1$ & 10.91 & 9.10 & 9.53 & 9.10 \\
\hline Average error & \multicolumn{2}{|c|}{72.56} & \multicolumn{2}{|c|}{} \\
\hline
\end{tabular}

\section{Conclusions}

This paper examined and analyzed leakage signals in different environments and proposed a new leak location method. First, the main frequency band of the leakage signal and the influence of noise on the positioning accuracy were analyzed. It was found that the accuracy of the time delay estimation was a key factor in positioning. On this basis, band-pass filters and BP neural networks were used to filter the out-of-band and in-band noises of the leakage signals, respectively. Finally, the generalized correlation method was used to estimate the time delay, and then the leakage location was achieved.

\section{Acknowledgements}

The authors are grateful to the National Science Foundation of China for its support of this research. This work was supported in part by the National Science Foundation of China under Grant 61461036 and 61761033.

\section{References}

[1] Ministry of Environment, Republic of Korea. 2015 Statistics of Waterworks. Available online: http://me.go.kr/ (accessed on 30 June2017).

[2] Martini, A., Troncossi, M., Rivola, A. (2015) Automatic Leak Detection in Buried Plastic Pipes 
of Water Supply Networks by Means of Vibration Measurements. Shock, 2015, 1-13.

[3] Zhang, Z., Tomita, E. J. (2002) Knocking Diagnostic using Wavelet Instantaneous Correlation Method. JSAE Review, 23, 443-449.

[4] Soldevila, Adrià, Blesa, Joaquim. (2016) Leak localization in water distribution networks using a mixed model-based data-driven approach. Control Engineering Practice, 55, 162-173.

[5] Ali, S., Ashraf, A., Qaisar, S. B., et. (2018) SimpliMote: A Wireless Sensor Network Monitoring Platform for Oil and Gas Pipelines. IEEE Sens. J., 12, 778-789.

[6] Gao, Y., Brennan, M. J., Joseph, P. F., Muggleton, J. M., and Hunaidi, O. (2004). A model of the correlation function of leak noise in buried plastic pipes. Journal of Sound \& Vibration, 277(1), 133-148.

[7] Delmas, J. P., Meurisse, Y. (2012) On the Cramer Rao bound and maximum likelihood in passive time delay estimation for complex signals. IEEE International Conference on Acoustics, Speech and Signal Processing, 22, 3541-3544.

[8] Hirose, A., Yoshida, S. (2012) Generalization characteristics of complex-valued feedforward neural networks in relation to signal coherence. IEEE Trans Neural Netw Learn Syst, 23, 541-551.

[9] Van, d. M. J. F., Jordaan, J. A. (2013) Comparison between general cross correlation and a template-matching scheme in the application of acoustic gunshot detection. Africon, 1-5.

[10] Matin, Thompson. Chapman, C. J., Howison, S. D., and Ockendon. J. R. (2001) Noise generation by water pipe leaks. 40th European Study Group with Industry.

[11] Adedeji, K. B., Hamam, Y., Abe, B. T., and Abu-Mahfouz, A. A. (2017) towards achieving a reliable leakage detection and localization algorithm for application in water piping networks: an overview. IEEE Access, 99, 1-1.

[12] Cataldo, A., Cannazza, G., Benedetto, E. D., and Giaquinto, N. (2012) A new method for detecting leaks in underground water pipelines. IEEE Sensors Journal, 12, 1660-1667.

[13] Li, Y., Li, T., Wang, K. (2011) A New Layer by Layer training algorithm for multilayer feedforward neural networks. International Conference on Advanced Computer Control, 600-603.

[14] Ionel, R., Ionel, S., and Ignea, A. (2010) Improved leak detection quality by automatic signal filtering. International Joint Conference on Computational Cybernetics and Technical Informatics, 425-430.

[15] Ahadi, M., Bakhtiar, M. S. (2010) Leak detection in water-filled plastic pipes through the application of tuned wavelet transforms to acoustic emission signals. Applied Acoustics, 71, 634639. 